\title{
Micro/Nanostructured Arrays: Fabrication, Applications, and Devices
}

\author{
Yue Li, ${ }^{1}$ Bingqiang Cao, ${ }^{2}$ Fengqiang Sun, ${ }^{3}$ and Sung $\mathrm{Oh} \mathrm{Cho}^{4}$ \\ ${ }^{1}$ Institute of Solid State Physics, Chinese Academy of Sciences, Hefei, Anhui 230031, China \\ ${ }^{2}$ School of Materials Science and Engineering, University of Jinan, Jinan, Shandong 250022, China \\ ${ }^{3}$ Department of Material Science and Engineering, South China Normal University, Guangzhou, Guangdong 510631, China \\ ${ }^{4}$ Department of Nuclear and Quantum Engineering, Korea Advanced Institute of Science and Technology (KAIST), \\ 373-1 Guseong-dong, Yuseong-gu, Daejeon 305-701, Republic of Korea
}

Correspondence should be addressed to Yue Li; yueli@issp.ac.cn

Received 2 July 2013; Accepted 2 July 2013

Copyright (C) 2013 Yue Li et al. This is an open access article distributed under the Creative Commons Attribution License, which permits unrestricted use, distribution, and reproduction in any medium, provided the original work is properly cited.

Recently, ordered micro/nanostructured arrays have attracted much interest due to their important applications in field emitters, nanogenerators, sensors, microfluidic devices, optoelectronic devices, and so forth. The traditional methods to fabricate micro/nanostructured arrays are generally based on lithographic techniques, for example, photolithography, electron beam lithography, ion beam lithography, and X-ray lithography, to name a few. However, these technologies are usually high costs and time consumption.

Therefore, scientists also endeavored to find other alternative strategies by facile routes with low costs. In this issue, S. Oh et al. and W.-W. Zhong et al. reported the selfassembly growth of one-dimensional oxide semiconductor nanostructures like $\mathrm{TiO}_{2}$ nanotube and $\mathrm{ZnO}$ nanorods. The influence of visible light irradiation and annealing on the photoluminescence and drug elution activity properties was investigated. G. Liu et al. reported the fabrication of selfstanding silver nanoplate arrays by seed-decorated electrochemical route using colloidal crystal as template. M. Veis and $\mathrm{R}$. Antos review the advances in optical and magnetooptical scatterometry of periodically ordered nanostructured arrays. $\mathrm{J}$. Hu et al. reported the metal surface plasmon resonance in periodic hexagonal lattice arrays of silver nanodisks, while E. J. Lee et al. reported the effect of liquid gallium on metal surfaces and characterized the morphology and chemical composition of metals heated in liquid gallium. As for device applications, Y. Takahashi et al. reported the multifunctional logic gate by means of nanodot array with different arrangements, and H. J. Kim et al. reported smallsized flat-tip CNT emitters for miniaturized X-ray tubes.

We believe that this specially organized issue could help readers to get more information on the fabrication of micro/ nanostructured arrays and their device applications.

Yue $\mathrm{Li}$ Bingqiang Cao Fengqiang Sun Sung Oh Cho 

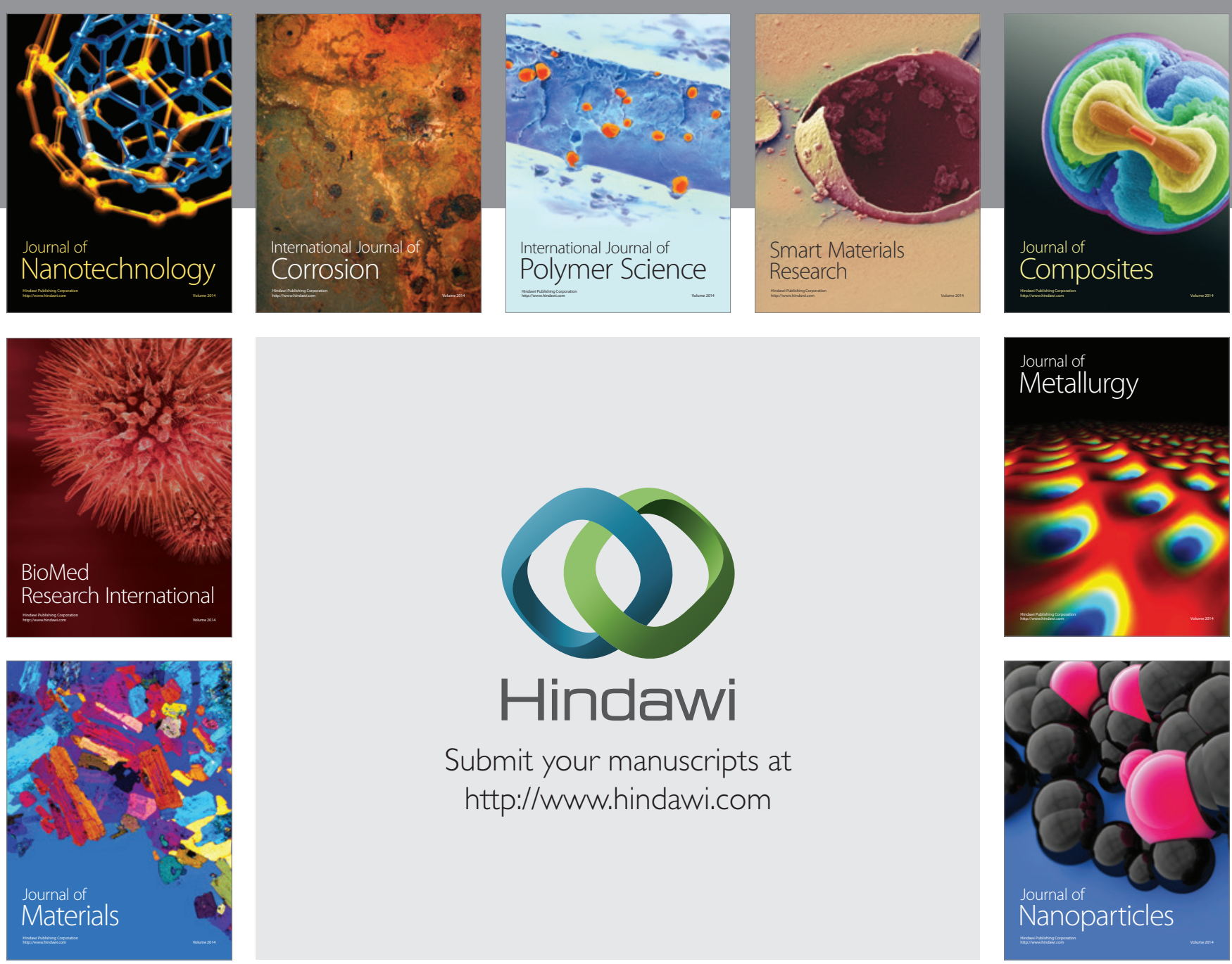

Submit your manuscripts at http://www.hindawi.com
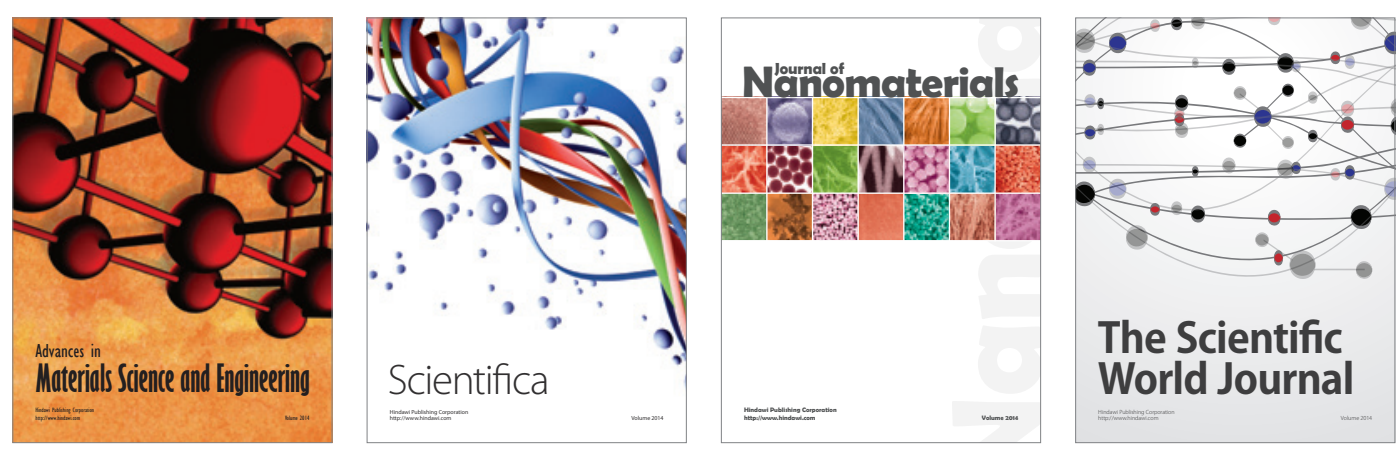

\section{The Scientific World Journal}
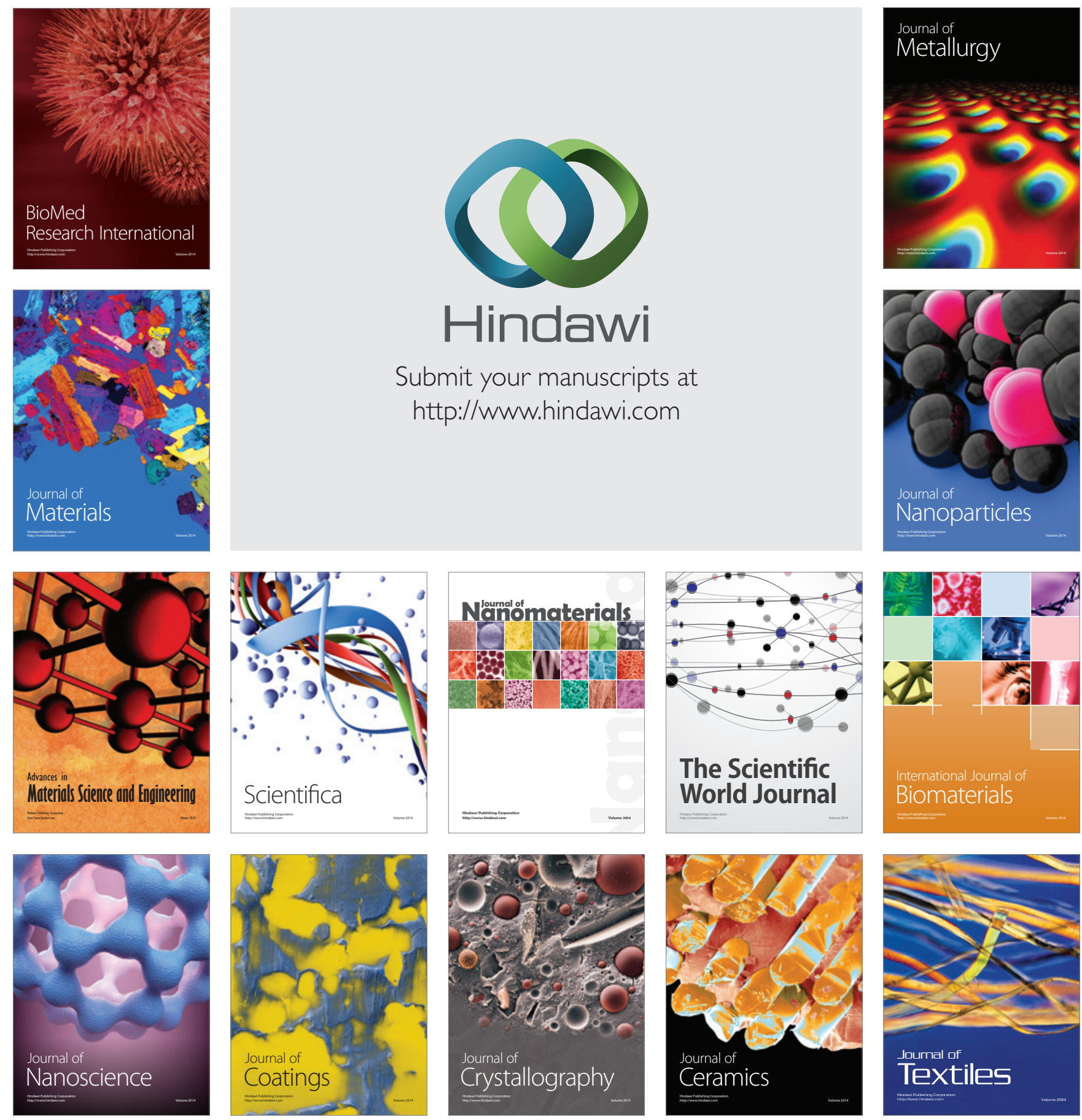